\title{
Kirsten Fudeman, Aaron Lawson, Carol Rosen and Devon Strolovitch (Editors), Cornell Working Papers in Linguistics, Romance Philology 17, Cornell Universitiy, Ithaca, NY 1999, 196 pagine
}

1. Sotto questo titolo è apparsa nell'autunno del 1999 un'antologia curata dai quattro editori, contenente 29 brani (ad opera dei curatori e altri collaboratori). Le linee direttrici sono esposte nella prefazione (Preface, 2 pagine introduttive fuori paginazione). La base è l'antologia Early Romance Texts: An Anthology (1980) di Rodney Sampson, definita invaluable, ma ormai di difficile accesso. Perciò, con la presente scelta di testi si cerca di ovviare a questa situazione. $\mathrm{Ci}$ sono però determinati limiti: infatti, sono stati tralasciati $i$ testi più noti, canonici, già bene studiati, ma nel contempo si è desistito anche dall'includere soltanto testi finora non pubblicati. In tal modo si è scelta una via di mezzo, includendo $i$ testi meno noti e meno discussi, tutti pubblicati, è vero, già prima ma in places now considered relatively obscure e parecchio tempo (perfino tutto un secolo) fa. La struttura di tutti i contributi è identica: ad una succinta introduzione (dati essenziali sul relativo testo) seguono il brano, i commenti, la bibliografia e la traduzione inglese. In tal modo l'antologia è coerente, di facile orientamento ed altrettanto facili confronti tra i singoli testi, il che è di notevole importanza scientifica e pedagogica. Infine, i curatori fanno risaltare il ruolo delle ricerche filologiche negli studi più propriamente linguistici.

2. Come detto, il volume racchiude 29 brani (i limiti della presente recensione impediscono di citare i singoli titoli e altri dati), divisi in cinque sezioni: I. Faith and Wisdom ["Fede e scienza" = trattati scientifici e morali; 9 brani, pp. 1-71]; II. Love Lyrics and The Love Bestiary ["Lirica e bestiari d'amore"; 7 brani, pp. 72-119]; III. More Beasts ["Ancora bestie" = altri bestiari; 7 brani, pp. 120-160]; IV. Property and Transactions ["Proprietà e Transazioni" = documenti amministrativo/giuridici e commerciali; 3 brani, pp. 161-174]; V. Cures and Concoctions ["Cure e decotti" = testi di medicina e affini; 3 brani, pp. 175196]. Sono rappresentati i testi dal 1200 al 1400, il che esclude il romeno. Tutti gli altri idiomi romanzi sono inclusi; da ovest ad est: il portoghese, il gallego, lo spagnolo, il catalano, il guascone, il provenzale, il francese, il retoromanzo (rappresentato dal Frammento di Einsiedeln), il dominio italiano (data l'epoca, rappresentato da quelli che dalla prospettiva odierna sono "dialetti", precisano gli autori; Prefazione) e il dalmatico. Oltre al romeno manca dunque anche il sardo, che nel periodo 1200-1400 è pur sempre già documentato. $\dot{E}$ relativamente alto il numero di testi in alfabeto ebraico, beninteso traslitterati ( 5 brani). I commenti linguistici sono succinti e, naturalmente, reciprocamente differenti, in dipendenza dai testi e dagli autori. Anche la bibliografia è assai diversa, variando da 32 titoli (per una traduzione spagnola di Brunetto Latini; 132-134) a uno solo (per di più risalente al 1841!) per il bestiario "Monosceros" di Philippe de Taun (159).

3. Osservazioni (tra parentesi le pagine): 1. (1-3): la forma ert (nel francese antico) è imperfetto nel v. 10 (della Vita di S. Giovanni), futuro invece nei vv. 33, 34, 69, 70, 82, 105 e 107 , risalente dunque a ERAT risp. ERIT, il che andrebbe commentato o per lo meno menzionato. - 2. (12): il catalano ullades non ha niente a che fare con l'ululare ma significa 
"sguardi, occhiate" ed è un derivato da ull "occhio", v. REW 6038. - 3. (36): non ci consta che nello spagnolo moderno esista il nome congedo, citato assieme all'omofono sostantivo italiano. - 4. (62): a proposito di plera (spagn. ant.) < PLORAT, dapprima si afferma che la grafia $p l$ (anziché $l l$ ) forse significa la non-palatalizzazione del nesso iniziale, poco dopo, invece, si dice che la vocale /e/ può essere dovuta alla palatalizazzione, sicché c'è una netta contraddizione. - 5. (76): il dittongo secondario nel franc. ant. conoistre dalla $/ \mathrm{J} /$ in sillaba chiusa non è uno sviluppo irregolare, dato che si tratta della fusione della vocale con la semivocale /y/ risultante dalla palatalizazzione di /sk/, come in CRESCERE > ${ }^{*}$ creistre $>$ croistre $>$ croître, NASCERE $>$ naistre $>$ naître, PASCERE $>$ paistre $>$ paître ecc. -6 . (77): l'evoluzione da CARUNCULA a *caronia ( $>$ franc. charogne, it. carogna ecc.) necessita di un commento, tanto più che né il REW né i dizionari italiani registrano CARUNCULA, sicché non risulta da dove l'autore (Josep Alba-Salas) abbia preso questa forma. - 7. (89): a proposito del rapporto companio - gahlaiba andrebbe discussa o almeno citata l'ipotesi di Günter Reichenkron, il quale, contrariamente alla spiegazione corrente, vede in gahlaiba un calco di COMPANIO, non viceversa, basandosi su tutta una serie di termini analoghi denotanti varie forme di associazioni nell'antichità romana, come COMBIBO, COMMILITO ecc. (G. Reichenkron, Historische Latein-Altromanische Grammatik I, Wiesbaden 1965, pp. 170-171). - 8. (97) non ci risulta nell'italiano standard convenente [citato come antiquato nello Zingarelli 1992] "event". - 9. (128): a proposito della forma verbale fallan "trovano" (in una traduzione castigliana di Brunetto Latini) l'autore (Josep Alba-Salas) suggerisce, citando J. Corominas, che la /f/ iniziale, qualificata di unetymological, rappresenti un'originaria $/ \mathrm{h} /$ proveniente dalla metatesi dell' $/ \mathrm{h} /$ interna dopo la palatalizazzione $/ \mathrm{fl}>\mathbb{I} /$, ma ci chiediamo, una volta avvenuta la palatalizzazione $/ \mathrm{fl}>\mathfrak{I} /$, da dove salta fuori la $/ \mathrm{h} /$ "interna"? - 10. (132): la /a/ (spagn. ant. aquen, aquende ecc.) non ci sembra proprio "misteriosa" e sopratutto non è unicamente avverbiale dato che l'abbiamo in aqui, allá, aquel, ant. aqueste ecc., nel romeno aici, acest, acel ecc.; si veda se non altro REW 4129, 4266, 4541, 4553. - 11. (145) il cammino da "per amore di" a "malgrado" (a proposito dell'it. ant. per mor de zo "malgrado questo") non è tanto "forzato" (stretch) quanto sembra credere Carol Rosen, perché troviamo un perfetto parallelo in sloveno, dove kljub temu significa "malgrado questo" e contiene il morfema lessicale ljub, ricorrente nel nome ljubezen "amore", nel verbo ljubiti "amare" ecc. - 12. (153): l'autrice Irene Mittelberg ammette per lo spagn. estrella una contaminazione STELLA > ASTRU, mentre Josep Alba-Salas (127) cita il Corominas che respinge quest'ipotesi dato che secondo lui ASTRU non era popolare in latino. Quale è dunque la spiegazione da preferire? - 13. (163): se nel brano riprodotto (un testamento trecentesco gallego) si legge ea "è", non è $e a$ written as $\langle e\rangle$ bensì semmai $\langle e\rangle$ written as ea. - 14. (167): fra i materiali in dalmatico trecentesco vorremmo vedere anche la nota lettera di Todru de Fomat indirizzata a ser Pon, unurivol canciler de Ragusa del 1325, che è più dalmatica di quella del 1397, qui riprodotta (cfr. Ž. Muljačić in P. Bec, Manuel pratique de philologie romane, II, Parigi 1971, p. 414). - 15 (ib.): il sintagma Pare me charisimu dovrebbe significare "Padre mio carissimo" (infatti, la lettera è firmata Vostiru fiol ecc.), sicché sorprende la traduzione It 
seems important to me. - 16. (169): deça, unito a dar, in un contratto stipulato a Traù/Trogir nel 1359, non risale a DECET e non significa "si addice" (it is fitting), ma è la variante settentrionale di deggia, oggi debba (<DEBEAT), e significa semplicemente "debba dare" (formula assai frequente in questo genere di testi antichi). - 17 (ib.): il croato grozde ["uva"] va corretto in grožđe; suoçivica non ha niente a che fare con civata "avena" (oats) né con suho "secco" (dry), ma è con tutta evidenza da identificarsi a sočivica "lente, lenticchia", derivato da sočivo, a sua volta tratto da sok (v. P. Skok, Dizionario etimol. croato e serbo, III, s. v.); quanto a sumisiça, "specie di grano secco" (some kind of dried grain), pur non potendo stabilire l'etimo per il momento, vi possiamo individuare lo stesso suffisso -ica, sicché va ovviamente eliminato *micata. - 18. (190): nella sequenza saber <*SAPE$\mathrm{RE}<\mathrm{SAPERE}$ mancano i diacritici di quantità, cioè saber $<*$ SAPERE $<$ SAPĔRE, senza i quali non risulta in che cosa consista la differenza tra le due forme latine.

4. Tra gli errori tipografici o comunque tecnici, tralasciando i più banali ed innocui, menzionamo i seguenti (la freccia punta verso la forma corretta): 1. (19): Crestomatie Romanica $\rightarrow$ Crestomație Romanică e Altfranzösische $\rightarrow$ Altfranzösisches (riferito a Übungsbuch). - 2. (63): per fuest "was" $\rightarrow$ "were" (così nella traduzione inglese); - 3. (72): per Segre $1947 \rightarrow 1957$ (cfr. la bibliografia, 74). - 4. (91): la forma bellazour non concorda con bellazor, nel testo (89). - 5. (105): Das Lieder $\rightarrow$ Das Lied o Die Lieder. - 6. (ib.): $\mathrm{XXII}^{\mathrm{e}}$ e XXIII $\rightarrow \mathrm{XII}^{\mathrm{e}}$ e XIII ${ }^{\mathrm{e}}$ risp. - 7. (113), r. 70: is an Aragonese $\rightarrow$ probabilm. as an Aragonese. - 8. (115): Muller $\rightarrow$ Müller e españoles (riferito a Lengua y literatura) $\rightarrow$ españolas. -9 . (132): vieillisement $\rightarrow$ vieillissement (s. v. charpentier). - 10. (144): tra palatal $[\lambda]$ e yod ci vuole una preposizione, probabilmente to. - 11. (148): nella riga 4: entao $\rightarrow$ então; - 12. (156): togliere le prime sei righe, che ripetono le ultime sei della pagina precedente. - 13. (170): Ugoli $\rightarrow$ Ugolini; - 14. (172): se il guasc. beno vale "vendette" (pass. rem. di "vendere"), non si tratta di loss of $/ \mathrm{n} /$ before $/ d /$ ma di loss of $/ d /$ after $/ \mathrm{n} /$. 15. (179 e 183) Valduz $\rightarrow$ Vaduz. - 16. (194), sub 46: CARMESIMUS $\rightarrow$ CARMESINUS.

5. L'antologia, qui recensita in modo alquanto sommario dato lo spazio a disposizione, è istruttiva, facilmente leggibile e maneggevole, limitata all'essenziale e interessante per la varietà di idiomi romanzi inclusi; insomma, varietas delectat, ossia il principio: relativamente molti testi rappresentati da brani piuttosto brevi (un po' come il Sermo Vulgaris Latinus di G. Rohlfs). Le mende, per lo più di lieve entità, potranno essere corrette senza problemi nelle edizioni ulteriori. Anche entro i limiti che gli autori si sono imposti, la loro scelta di testi romanzi antichi avrà il suo posto negli studi dei primi passi degli idiomi romanzi occidentali. 